\title{
On Stability of Vector Nonlinear Integrodifferential Equations
}

\author{
Michael Gil’ \\ Department of Mathematics, Ben-Gurion University of the Negev, P.O. Box 653, 84105 Beersheba, Israel \\ Correspondence should be addressed to Michael Gil'; gilmi@bezeqint.net
}

Received 15 March 2016; Accepted 5 May 2016

Academic Editor: Josè A. Tenereiro Machado

Copyright (C) 2016 Michael Gil'. This is an open access article distributed under the Creative Commons Attribution License, which permits unrestricted use, distribution, and reproduction in any medium, provided the original work is properly cited.

Let $\Omega$ be a bounded domain in a real Euclidean space. We consider the equation $\partial u(t, x) / \partial t=C(x) u(t, x)+\int_{\Omega} K(x, s) u(t, s) d s+$ $[F(u)](t, x)(t>0 ; x \in \Omega)$, where $C(\cdot)$ and $K(\cdot, \cdot)$ are matrix-valued functions and $F(\cdot)$ is a nonlinear mapping. Conditions for the exponential stability of the steady state are established. Our approach is based on a norm estimate for operator commutators.

\section{Introduction and Statement of the Main Result}

Throughout this paper, $\mathbb{C}^{n}$ is the complex $n$-dimensional Euclidean space with a scalar product $(\cdot, \cdot)_{n}$ and norm $\|\cdot\|_{n}$ $=\sqrt{(\cdot, \cdot)_{n}} ; \mathbb{C}^{n \times n}$ is the set of $n \times n$-matrices; $I$ is the unit operator in corresponding space; $\Omega$ is a bounded domain with a smooth boundary in a real Euclidean space; $L^{2}\left(\mathbb{C}^{n}, \Omega\right)=L^{2}$ is the Hilbert space of functions defined on $\Omega$ with values in $\mathbb{C}^{n}$, the scalar product

$$
(v, w)_{L^{2}}=\int_{\Omega}(v(x), w(x))_{n} d x \quad\left(w, v \in L^{2}\right),
$$

and the norm $\|\cdot\|_{L^{2}}=\sqrt{(\cdot, \cdot)_{L^{2}}}$.

Our main object in this paper is the equation

$$
\begin{aligned}
\frac{\partial u(t, x)}{\partial t}= & C(x) u(t, x)+\int_{\Omega} K(x, s) u(t, s) d s \\
& +[F(u)](t, x) \quad(t>0 ; x \in \Omega),
\end{aligned}
$$

where $C(\cdot)$ and $K(\cdot, \cdot)$ are matrix-valued functions defined on $\Omega$ and $\Omega \times \Omega$, respectively, with values in $\mathbb{C}^{n \times n}$, and $F(\cdot)$ : $L^{2} \rightarrow L^{2}$ satisfy conditions pointed out below, and $u(\cdot, \cdot)$ is unknown.

Traditionally, (2) is called the Barbashin type integrodifferential equation or simply the Barbashin equation. It plays an essential role in numerous applications, in particular, in kinetic theory [1], transport theory [2], continuous mechanics [3], control theory [4], radiation theory [5, 6], and the dynamics of populations [7]. Regarding other applications, see [8]. The classical results on the Barbashin equation are represented in the well-known book [9]. The recent results about various aspects of the theory of the Barbashin equation can be found, for instance, in [10-14] and the references given therein. In particular, in [11], the author investigates the solvability conditions for the Cauchy problem for a Barbashin equation in the space of bounded continuous functions and in the space of continuous vector-valued functions with the values in an ideal Banach space. The stability and boundedness of solutions to a linear scalar nonautonomous Barbashin equation have been investigated in [15].

The literature on the asymptotic properties of integrodifferential equations is rather rich (cf. [16-22] and the references given therein), but the stability of nonlinear vector integrodifferential equations is almost not investigated. It is at an early stage of development.

A solution of $(2)$ is a function $u(t, \cdot):[0, \infty) \rightarrow L^{2}$ having a measurable derivative bounded on each finite interval.

It is assumed that under consideration $F$ provides the existence and uniqueness of solutions (e.g., it is Lipschitz continuous). The zero solution of (2) is said to be exponentially stable, if there are constants $m_{0} \geq 1, \delta_{0}>0$, and $\alpha>0$, such that $\|u(t)\|_{L^{2}} \leq m_{0}\|u(0)\|_{L^{2}} e^{-\alpha t}(t \geq 0)$, provided $\|u(0)\|_{L^{2}} \leq$ $\delta_{0}$. It is globally exponentially stable if $\delta_{0}=\infty$.

Suppose that, for a positive $r \leq \infty$,

$$
\|F(h)\|_{L^{2}} \leq q\|h\|_{L^{2}} \quad\left(h \in L^{2} ;\|h\|_{L^{2}} \leq r\right) .
$$


For example, for an integer $p>1$, let $[F(h)](x)=(T h(x))^{p}$. Here,

$$
T h(x)=\int_{\Omega} b(x, s) h(s) d s
$$

with a matrix kernel $b(x, s)$ satisfying

$$
J_{p}=\left(\int_{\Omega}\left(\int_{\Omega}\|b(x, s)\|_{n}^{2} d s\right)^{p} d x\right)^{1 / 2}<\infty .
$$

Then, by the Schwarz inequality,

$$
\|T h(x)\|_{n}^{2} \leq \int_{\Omega}\|b(x, s)\|_{n}^{2} d s\|h\|_{L^{2}}^{2} .
$$

Thus,

$$
\begin{aligned}
& \int_{\Omega}\|[F(h)](x)\|^{2} d x \\
& \leq \int_{\Omega}\left(\int_{\Omega}\|b(x, s)\|_{n}^{2} d s\right)^{p} d x\|h\|_{L^{2}}^{2 p} .
\end{aligned}
$$

Hence, for any $r<\infty$, we have condition (3) with $q=r^{p-1} J_{p}$.

The following notations are introduced: for a linear operator $A, A^{*}$ is the adjoint operator, $\|A\|$ is the operator norm, and $\sigma(A)$ is the spectrum. For $n \times n$-matrix $C$, put

$$
g(C)=\left[N_{2}^{2}(C)-\sum_{k=1}^{n}\left|\lambda_{k}(C)\right|^{2}\right]^{1 / 2},
$$

where $\lambda_{k}(C), k=1, \ldots, n$, are the eigenvalues of $C$, counted with their multiplicities; $N_{2}(C)=\left(\text { Trace } C C^{*}\right)^{1 / 2}$ is the Frobenius (Hilbert-Schmidt) norm of $C$. The following relations are checked in [23, Section 2.1]: $g^{2}(C) \leq N_{2}^{2}(C)-$ |Trace $C^{2}$,

$$
\begin{gathered}
g\left(e^{i \tau} C+z I\right)=g(C) \quad(\tau \in \mathbb{R}, z \in \mathbb{C}), \\
g^{2}(C) \leq \frac{N_{2}^{2}\left(C-C^{*}\right)}{2} .
\end{gathered}
$$

If $C$ is a normal matrix, $C C^{*}=C^{*} C$, then $g(C)=0$. Furthermore, denote

$$
\begin{aligned}
\xi:= & \frac{1}{2} \sup _{h \in L^{2},\|h\|_{L^{2}}=1} \int_{\Omega} \int_{\Omega}\left(\left(K(x, s)+K^{*}(s, x)\right) h(s),\right. \\
& h(x))_{n} d s d x \\
\gamma= & \left(\int_{\Omega} \int_{\Omega}\|C(x) K(x, s)-K(x, s) C(s)\|_{n}^{2} d s d x\right)^{1 / 2}
\end{aligned}
$$

and assume that

$$
\begin{aligned}
\alpha_{0} & :=\xi+\sup _{x} \Re \sigma(C(x))=\xi+\sup _{x} \max _{k} \Re \lambda_{k}(C(x)) \\
& <0 .
\end{aligned}
$$

In addition, with the notation $g_{0}=\sup _{x} g(C(x))$, put

$$
\begin{aligned}
\chi & =\sum_{j, k=0}^{n-1} \frac{g_{0}^{j+k}(k+j) !}{2^{j+k}\left|\alpha_{0}\right|^{j+k+1}(j ! k !)^{3 / 2}}, \\
p(t) & =\sum_{k=0}^{n-1} \frac{t^{k} g_{0}^{k}}{(k !)^{3 / 2}}, \\
\zeta_{0} & =2 \int_{0}^{\infty} e^{2 \alpha_{0} t} p(t) \int_{0}^{t} p(t-s) p(s) d s d t .
\end{aligned}
$$

This integral is simply calculated. If $C(x)$ is a normal matrix for all $x$, then

$$
\begin{aligned}
g(C(x)) & =0, \\
p(t) & \equiv 1, \\
\chi & =\frac{1}{\left|\alpha_{0}\right|}, \\
\zeta_{0} & =\frac{1}{2\left|\alpha_{0}\right|^{2}} .
\end{aligned}
$$

Now, we are in a position to formulate our main result.

Theorem 1. Let conditions (3), (11), and

$$
\gamma \zeta_{0}+\chi q<1
$$

hold. Then, the zero solution to (2) is exponentially stable. If, in addition, $r=\infty$ in (3), then the zero solution is globally exponentially stable.

This theorem is proved in the next 3 sections. It gives us "good" results when $\gamma$ is "small," that is, if matrices $K(x, s)$ and $C(x)$ "almost commute" and $\sup _{x, s}\|C(x)-C(s)\|_{n}$ is "small." If (2) is scalar, then $g_{0}=0$,

$$
\begin{aligned}
\chi & =\frac{1}{\left|\alpha_{0}\right|}, \\
\gamma & =0, \\
p(t) & \equiv 1, \\
\zeta_{0} & =\frac{1}{2\left|\alpha_{0}\right|^{2}} .
\end{aligned}
$$

So, in the scalar case, condition (14) takes the form

$$
q<\left|\alpha_{0}\right|
$$

This condition is similar to the stability test derived in [24] for scalar integrodifferential equations.

\section{Auxiliary Results}

Let $\mathscr{H}$ be a Hilbert space with a scalar product $(\cdot, \cdot)_{\mathscr{H}}$ and the norm $\|\cdot\|_{\mathscr{H}}=\sqrt{(\cdot, \cdot)_{\mathscr{H}}} ; \mathscr{B}(\mathscr{H})$ denotes the set of bounded linear operators in $\mathscr{H}$ and $\left[A_{1}, A_{2}\right]=A_{1} A_{2}-A_{2} A_{1}$ is the commutator of $A_{1}, A_{2} \in \mathscr{B}(\mathscr{H})$. 
Lemma 2. Let $A, B \in \mathscr{B}(\mathscr{H})$ and $C=[A, B]$. Then,

$$
\left[e^{A t}, B\right]=\int_{0}^{t} e^{A s} C e^{A(t-s)} d s .
$$

Proof. Put $J(t)=\int_{0}^{t} e^{A s} C e^{A(t-s)} d s$. Then, $(d / d t)\left(J(t) e^{-t A}\right)=$ $e^{A t} C e^{-A t}$. On the other hand,

$$
\frac{d}{d t}\left(\left[e^{A t}, B\right] e^{-t A}\right)=\frac{d}{d t}\left(e^{A t} B e^{-A t}-B\right)=e^{A t} C e^{-A t} .
$$

So, $\left[e^{A t}, B\right]=J$, as claimed.

Let

$$
\alpha(A):=\sup \Re \sigma(A)<0 .
$$

Then, the Lyapunov equation

$$
W A+(W A)^{*}=-2 I
$$

has a unique solution $W \in \mathscr{B}(\mathscr{H})$ and it can be represented as

$$
W=2 \int_{0}^{\infty} e^{A^{*} t} e^{A t} d t
$$

(cf. [25]). Denote $\Lambda_{B}=(1 / 2) \sup \sigma\left(B+B^{*}\right)$,

$$
\begin{gathered}
\zeta(A)=2 \int_{0}^{\infty}\left\|e^{A t}\right\| \int_{0}^{t}\left\|e^{A s}\right\|\left\|e^{A(t-s)}\right\| d s d t, \\
\psi(W, B)= \begin{cases}\Lambda_{B}\|W\| & \text { if } \Lambda_{B}>0, \\
\Lambda_{B} \lambda_{W} & \text { if } \Lambda_{B} \leq 0,\end{cases}
\end{gathered}
$$

where $\lambda_{W}=\inf \sigma(W)$.

Lemma 3. Under condition (19), one has

$$
\begin{aligned}
\operatorname{Re}(W B) & =\frac{1}{2}\left((W B)+(W B)^{*}\right) \\
& \leq(\psi(W, B)+\|C\| \zeta(A)) I .
\end{aligned}
$$

Proof. Making use of (21), we can write

$$
\operatorname{Re}(W B)=\int_{0}^{\infty}\left(e^{A^{*} t} e^{A t} B+B^{*} e^{A^{*} t} e^{A t}\right) d t
$$

But $e^{A t} B=B e^{A t}+\left[e^{A t}, B\right], B^{*} e^{A^{*} t}=e^{A^{*} t} B^{*}+\left[B^{*}, e^{A^{*} t}\right]=$ $e^{A^{*} t} B^{*}+\left[e^{A t}, B\right]^{*}$. So $2 \operatorname{Re}(W B)=J_{1}+J_{2}$, where

$$
\begin{aligned}
& J_{1}=\int_{0}^{\infty} e^{A^{*} t}\left(B+B^{*}\right) e^{A t} d t \\
& J_{2}=\int_{0}^{\infty}\left(e^{A^{*} t}\left[e^{A t}, B\right]+\left(e^{A^{*} t}\left[e^{A t}, B\right]\right)^{*}\right) d t .
\end{aligned}
$$

We have

$$
J_{1} \leq 2 \Lambda_{B} \int_{0}^{\infty} e^{A^{*} t} e^{A t} d t=\Lambda_{B} W
$$

If $\Lambda_{B}>0$, then $J_{1} \leq \Lambda_{B}\|W\| I$. If $\Lambda_{B}<0$, then $J_{1} \leq \Lambda_{B} \lambda_{W} I$. So $J_{1} \leq 2 \psi(W, B) I$.

In addition, by Lemma 2 ,

$$
\begin{aligned}
\left\|J_{2}\right\| & \leq 2 \int_{0}^{\infty}\left\|e^{A t}\right\|\left\|\left[e^{A t}, B\right]\right\| d t \\
& \leq 2 \int_{0}^{\infty}\left\|e^{A t}\right\|\|C\| \int_{0}^{t}\left\|e^{A s}\right\|\left\|e^{A(t-s)}\right\| d s d t \\
& =\|C\| \zeta(A) .
\end{aligned}
$$

This proves the lemma.

\section{Equations in a Hilbert Space}

In this section, for simplicity, we put $\|\cdot\|_{\mathscr{H}}=\|\cdot\|$. Put $\omega(r)=$ $\{v \in \mathscr{H},\|v\| \leq r\}(0<r \leq \infty)$. Consider in $\mathscr{H}$ the equation

$$
\frac{d}{d t} u=(A+B) u+F(u) \quad(t \geq 0)
$$

where $A, B \in \mathscr{B}(\mathscr{H})$ and $F$ continuously maps $\omega(r)$ into $\mathscr{H}$ and satisfies

$$
\|F v\| \leq q\|v\| \quad(v \in \omega(r)) .
$$

The solution and stability are defined as in Section 1. The existence and uniqueness of solutions are assumed. Recall that $W$ is a solution of (20).

Lemma 4. Let conditions (19) and (29) with $r=\infty$ hold. Then, any solution of (28) satisfies the inequality

$$
\|u(t)\| \leq\left(\frac{\|W\|}{\lambda_{W}}\right)^{1 / 2}\|u(0)\| e^{-v t}, \quad t \geq 0,
$$

$$
\text { where } v:=1-\psi(W, B)-\zeta(A)\|C\|-q\|W\| \text {. }
$$

Proof. For brevity, we write $[F u](t)=F u(t)$. Multiplying (28) by $W$ and doing the scalar product, we get

$$
\begin{aligned}
\left(W u^{\prime}(t), u(t)\right)= & (W(A+B) u(t), u(t)) \\
& +(W F u(t), u(t))
\end{aligned}
$$

Since $(d / d t)(W u(t), u(t))=\left(W u^{\prime}(t), u(t)\right)+\left(u(t), W u^{\prime}(t)\right)$, due to (20) and Lemma 3, it can be written that

$$
\begin{aligned}
\frac{d}{d t}(W u(t), u(t)) \\
=2 \operatorname{Re}(W(A+B) u(t), u(t)) \\
\quad+2 \operatorname{Re}(W F u(t), u(t)) \\
\leq 2(-1+\psi(W, B)+\zeta(A)\|C\|)(u(t), u(t)) \\
\quad+2 \operatorname{Re}(W F u(t), u(t)) .
\end{aligned}
$$

Taking into account the fact that due to (29)

$$
\begin{aligned}
(W F u(t), u(t)) & \leq\|W\|\|F u(t)\|\|u(t)\| \\
& \leq\|W\| q\|u(t)\|^{2}
\end{aligned}
$$


we get

$$
\frac{d}{d t}(W u(t), u(t)) \leq-2 v(u(t), u(t))
$$

From this inequality, we have $(W u(t), u(t)) \leq(W u(0)$, $u(0)) e^{-2 v t}$. Hence,

$$
\lambda_{W}(u(t), u(t)) \leq\|W\|(u(0), u(0)) e^{-2 v t},
$$

as claimed.

Lemma 5. Let conditions (29) and $v<0$ hold. Then, the zero solution to (28) is exponentially stable. If $r=\infty$ in (29), then the zero solution to (28) is globally exponentially stable.

Proof. If $r=\infty$, then the required result is due to the previous lemma. If $r<\infty$, then, taking $\|u(0)\|<r\left(\lambda_{W} /\|W\|\right)^{1 / 2}$ due to the previous lemma, $\|u(t)\|<r$. Hence, we easily obtain the required result.

\section{Proof of Theorem 1}

Take

$$
\begin{aligned}
& A h(x)=(C(x)+\xi I) h(x), \\
& B h(x)=\int_{\Omega} K(x, s) h(s) d s-\xi h(x)
\end{aligned}
$$

$$
\left(h \in L^{2}\right)
$$

Then, $\Lambda(B) \leq 0$ and

$$
\begin{aligned}
& {[A, B] h(x)} \\
& \quad=\int_{\Omega}[C(x) K(x, s)-K(x, s) C(s)] h(s) d s .
\end{aligned}
$$

So $\|[A, B]\|_{L^{2}} \leq \gamma$. Due to [23, Example 1.7.3],

$$
\left\|e^{C(x) t}\right\|_{n} \leq e^{\alpha(C(x)) t} \sum_{k=0}^{n-1} \frac{t^{k} g^{k}(C(x))}{(k !)^{3 / 2}} \quad(t \geq 0),
$$

where $\alpha(C(x))=\sup \operatorname{Re} \sigma(C(x))$. Hence,

$$
\left\|e^{A t}\right\|_{L^{2}} \leq \sup _{x}\left\|e^{(C(x)+\xi) t}\right\|_{n} \leq e^{\alpha_{0} t} p(t) \quad(t \geq 0),
$$

since $g(C(x)+\xi I)=g(C(x))$. Consequently, $\zeta(A) \leq \zeta_{0}$. In addition,

$$
\|W\|_{L^{2}} \leq 2 \int_{0}^{\infty}\left\|e^{A t}\right\|_{L^{2}}^{2} d t \leq 2 \int_{0}^{\infty} e^{2 \alpha_{0} t} p^{2}(t) d t=\chi .
$$

Now, the required result is due to Lemma 5.

\section{Competing Interests}

The author declares that there are no competing interests regarding the publication of this paper.

\section{References}

[1] C. Cercignani, Mathematical Methods in Kinetic Theory, Macmillian, New York, NY, USA, 1969.

[2] H. G. Kaper, C. G. Lekkerkerker, and J. Hejtmanek, Spectral Methods in Linear Transport Theory, vol. 5 of Operator Theory: Advances and Applications, Birkhäuser, Basel, Switzerland, 1982.

[3] V. M. Aleksandrov and E. V. Kovalenko, Problems in Continuous Mechanics with Mixed Boundary Conditions, Nauka, Moscow, Russia, 1986 (Russian).

[4] A. L. Khoteev, "An optimal control problem for integrodifferential equations of Barbashin type," in Problemy Optimizacii Upravlenija, pp. 74-87, Minsk, Russia, 1976 (Russian).

[5] K. M. Case and P. F. Zweifel, Linear Transport Theory, AddisonWesley, Reading, Mass, USA, 1967.

[6] G. I. Marchuk, The Methods of Calculation for Nuclear Reactors, Atomizdat, Moscow, Russia, 1961 (Russian).

[7] H. R. Thieme, A Differential-Integral Equation Modelling the Dynamics of Populations with a Rank Structure, vol. 68 of Lecture Notes in Biomathematics, 1986.

[8] A. W. England, "Thermal microwave emission from a halfspace containing scatterers," Radio Science, vol. 9, no. 4, pp. 447-454, 1974.

[9] J. M. Appell, A. S. Kalitvin, and P. P. Zabrejko, Partial Integral Operators and Integro-Differential Equations, Marcel Dekker, New York, NY, USA, 2000.

[10] A. S. Kalitvin, "On two problems for the Barbashin integrodifferential equation," Journal of Mathematical Sciences, vol. 126, no. 6, pp. 1600-1606, 2005.

[11] A. S. Kalitvin, "Some aspects of the theory of integro-differential Barbashin equations in function spaces," Journal of Mathematical Sciences, vol. 188, no. 3, pp. 241-249, 2013.

[12] B. G. Pachpatte, "On a parabolic type Fredholm integrodifferential equation," Numerical Functional Analysis and Optimization, vol. 30, no. 1-2, pp. 136-147, 2009.

[13] B. G. Pachpatte, "On a nonlinear Volterra integral equation in two variables," Sarajevo Journal of Mathematics, vol. 6, no. 19, pp. 59-73, 2010.

[14] B. G. Pachpatte, "On a parabolic integrodifferential equation of Barbashin type," Commentationes Mathematicae Universitatis Carolinae, vol. 52, no. 3, pp. 391-401, 2011.

[15] M. Gil,' "On stability of linear Barbashin type integrodifferential equations," Mathematical Problems in Engineering, vol. 2015, Article ID 962565, 5 pages, 2015.

[16] R. P. Agarwal, A. Domoshnitsky, and Y. Goltser, "Stability of partial functional integro-differential equations," Journal of Dynamical and Control Systems, vol. 12, no. 1, pp. 1-31, 2006.

[17] N. M. Chuong, T. D. Ke, and N. N. Quan, "Stability for a class of fractional partial integro-differential equations," Journal of Integral Equations and Applications, vol. 26, no. 2, pp. 145-170, 2014.

[18] A. Domoshnitsky and Y. M. Goltser, "Approach to study of bifurcations and stability of integro-differential equations," Mathematical and Computer Modelling, vol. 36, no. 6, pp. 663678, 2002.

[19] A. D. Drozdov and M. I. Gil, "Stability of linear integrodifferential equations with periodic coefficients," Quarterly of Applied Mathematics, vol. 54, no. 4, pp. 609-624, 1996.

[20] Ya. Goltser and A. Domoshnitsky, "Bifurcations and stability of integro-differential equations," Nonlinear Analysis: Theory, Methods \& Applications, vol. 47, no. 2, pp. 953-967, 2001. 
[21] J. Cao and Z. Huang, "Existence and exponential stability of weighted pseudo almost periodic classical solutions of integrodifferential equations with analytic semigroups," Differential Equations and Dynamical Systems, vol. 23, no. 3, pp. 241-256, 2015.

[22] N. T. Dung, "On exponential stability of linear Levin-Nohel integro-differential equations," Journal of Mathematical Physics, vol. 56, Article ID 022702, 2015.

[23] M. I. Gil', Operator Functions and Localization of Spectra, vol. 1830 of Lecture Notes in Mathematics, Springer, Berlin, Germany, 2003.

[24] M. I. Gil', "Stability of Fredholm type integro-parabolic equations," Journal of Mathematical Analysis and Applications, vol. 244, no. 2, pp. 318-332, 2000.

[25] L. Daleckii and M. G. Krein, Stability of Solutions of Differential Equations in Banach Space, American Mathematical Society, Providence, RI, USA, 1974. 


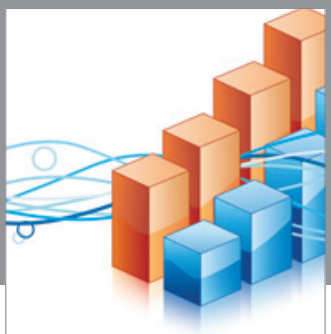

Advances in

Operations Research

vatem alat4

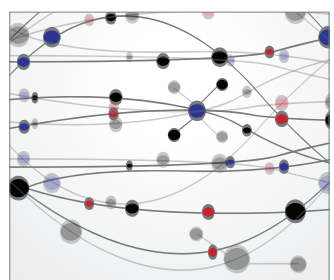

\section{The Scientific} World Journal
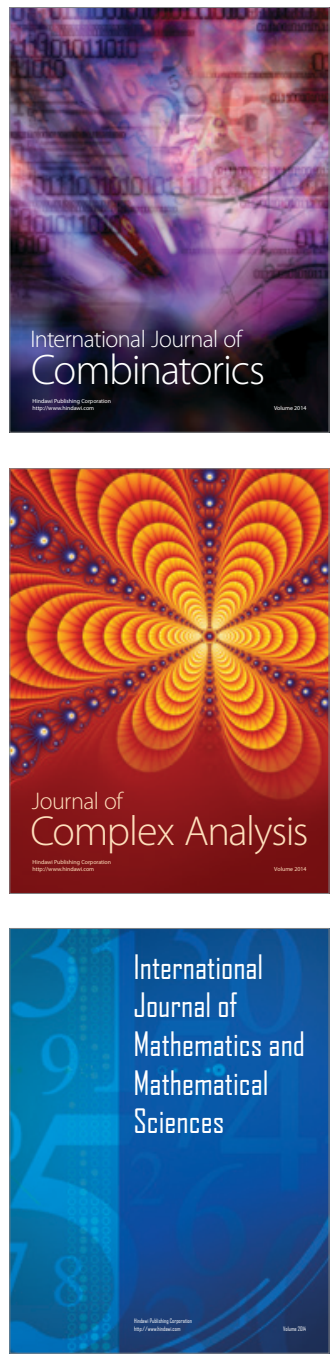
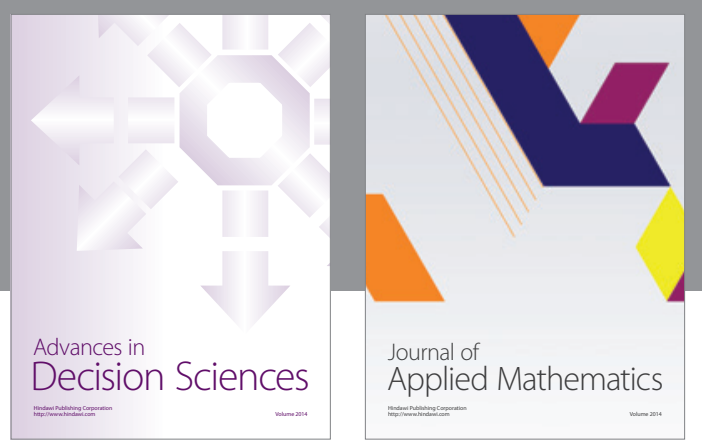

Algebra

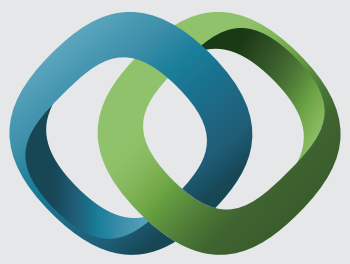

\section{Hindawi}

Submit your manuscripts at

http://www.hindawi.com
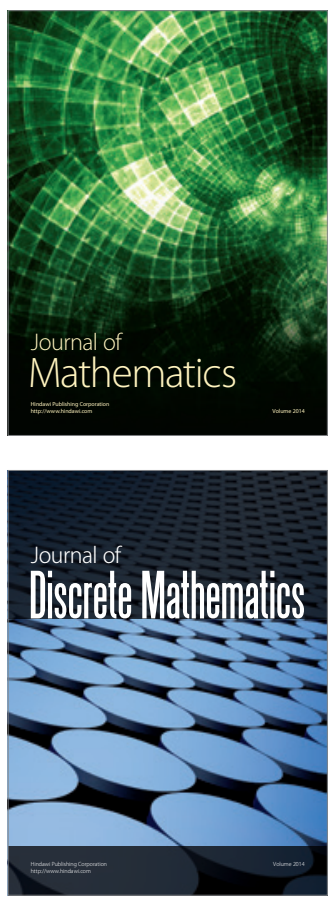

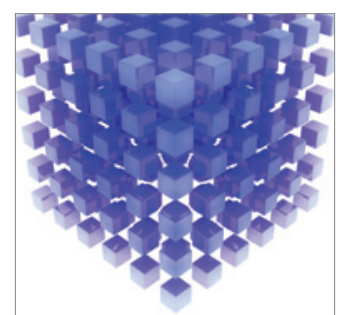

Mathematical Problems in Engineering
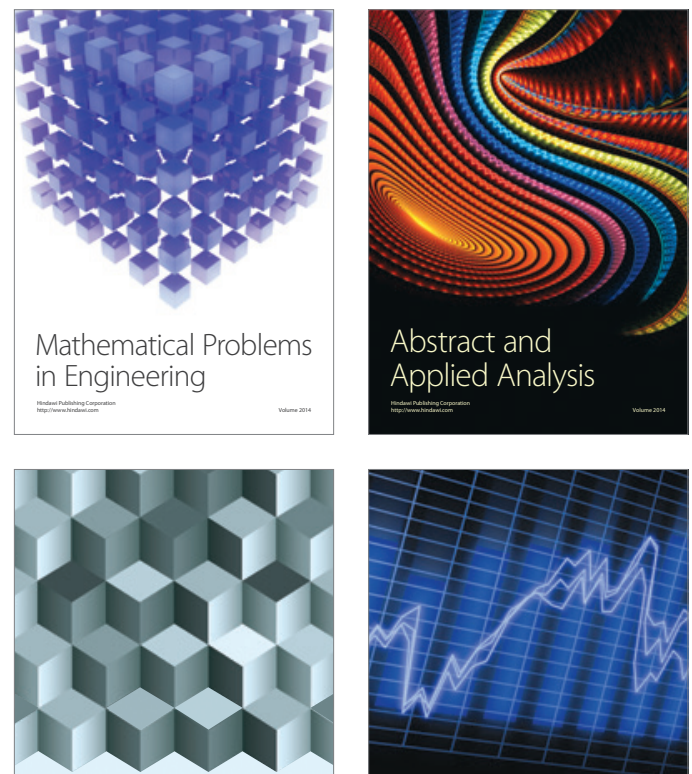

Journal of

Function Spaces

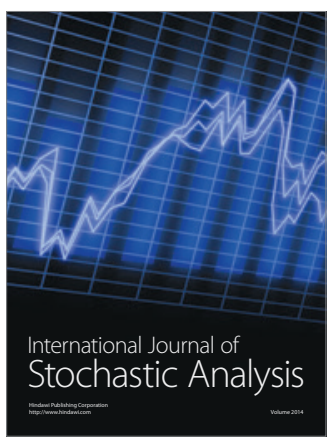

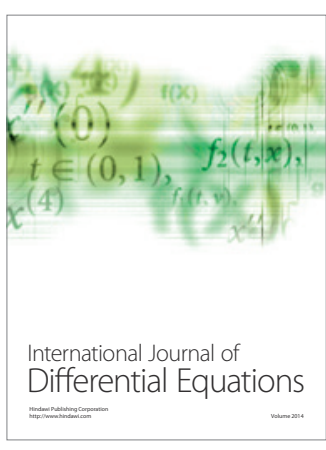
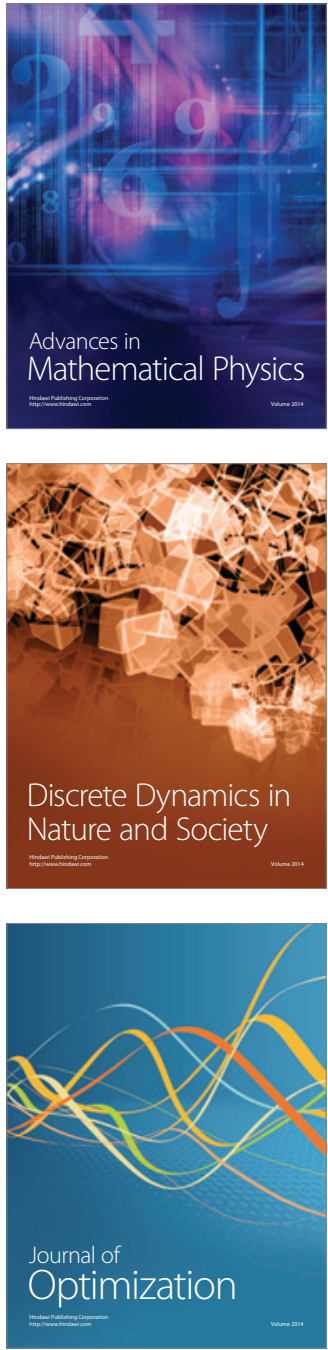